\title{
Spatial classification of glaucomatous visual field loss
}

\author{
David B Henson, Susan E Spenceley, David R Bull
}

\begin{abstract}
Aims-To develop and describe an objective classification system for the spatial patterns of visual field loss found in glaucoma.

Methods-The 560 Humphrey visual field analyser (program 24-2) records were used to train an artificial neural network (ANN). The type of network used, a Kohonen self organising feature map (SOM), was configured to organise the visual field defects into 25 classes of superior visual field loss and 25 classes of inferior visual field loss. Each group of 25 classes was arranged in a 5 by 5 map.

Results-The SOM successfully classified the defects on the basis of the patterns of loss. The maps show a continuum of change as one moves across them with early loss at one corner and advanced loss at the opposite corner.

Conclusions-ANNs can classify visual field data on the basis of the pattern of loss. Once trained the ANN can be used to classify longitudinal visual field data which may prove valuable in monitoring visual field loss.
\end{abstract}

(Br F Ophthalmol 1996;80:526-531)

Glaucoma is known to produce characteristic patterns of visual field loss. Aulhorn and Harms $^{1}$ classified 954 eyes with circumscribed defects in the central visual field into eight different groups. The subjective classification being based upon the location, shape, and extent of loss. Similar, descriptive classifications have been given by Aulhorn, ${ }^{2}$ Aulhorn and Karmeyer, ${ }^{3}$ Drance, ${ }^{4}$ and, as part of an investigation into progressive loss, by both Hart and Becker ${ }^{5}$ and Jay and Murdoch. ${ }^{6}$

These subjective classification systems, which are all based upon kinetic data, have a number of shortcomings. The individual classes often lack precise definitions which can lead to problems of misclassification. The number of classes are invariably small, which limits their usefulness in grading the extent of loss and in monitoring progression. Finally, the classes are often based upon preconceived notions concerning the nature of glaucomatous loss.

Artificial neural networks (ANNs), which are capable of learning from examples, possess a series of attributes that make them particularly useful in the tasks of differentiating and classifying spatial patterns. They have an ability to learn similarities among patterns directly from instances of them, they are capable of using all the data within very large data sets, they have no preconceived notions, they have an ability to generalise when used to process previously unseen data, and they can be used to classify the results from static strategies, such as the 24-2 program of the Humphrey visual field analyser (HVA).

There are two broad classes of ANNsthose which use supervised learning and those which use unsupervised learning. The supervised ANN learns from a training set of data in which each item has already been classified. It then learns to reproduce this classification. The back propagation network, ${ }^{7}$ which has been used by a number of researchers interested in developing automated systems for the recognition and classification of visual field defects, ${ }^{8-14}$ is typical of this type of learning.

The unsupervised $\mathrm{ANN}$ does not require the training data to be classified. The classification is performed by the network. The Kohonen self organising feature map (SOM) ${ }^{15}$ is typical of an unsupervised method of learning. One of the major advantages of the SOM is that it overcomes the criticism that ANNs simply learn to duplicate the preconceived judgments of the data classifier(s).

Because ANNs learn from their training set examples the composition of this set becomes an important variable in the final outcome. ${ }^{12}$ In the case of the SOM the percentage of output nodes allocated to a particular group of defects will be roughly in proportion to the number of cases within the training set. If, for example, the training set had a large number of eyes with early visual field loss and very few with advanced loss then the resulting network would allocate a large number of the output nodes to the early loss examples and few to the advanced ones. A balance of defects within the training set is important if it is to represent accurately all the types of loss found within the population.

The spatial patterns of loss within the superior and inferior hemifields show a degree of independence due to the anatomical arrangement of visual fibres at the optic nerve head. When spatially classifying the whole visual field this arrangement can result in a certain number of redundant classes in which different combinations of the same the superior and inferior hemifield patterns occur. This problem can be overcome by classifying independently the superior and inferior hemifields.

This paper describes an objective classification system for the spatial patterns of visual field loss found in glaucoma. The classification is based upon the output of a SOM trained with data from the 24-2 program of the HVA. The superior and inferior fields are classified independently. This paper also highlights how 
the classification system is dependent upon the inclusion criteria of the training set.

\section{Materials and methods}

TRAINING DATA

The training set pool (560 records from 560 patients) was selected from a database of visual field records collected at Moorfields Eye Hospital and the Department of Ophthalmology, University of Dalhousie. The data were collected with an HVA either as part of the routine care of patients in a glaucoma clinic or as part of a longitudinal project looking into progressive visual field loss.

The test locations used were a subset of those in the HVA 24-2 program (see Fig 1). Excluded locations were the two that normally fall within the blind spot region and the two that are beyond an eccentricity of 24 degrees in the temporal field. This subset contained 25 test locations in both the superior and inferior hemifield. Data from left eyes were flipped around the vertical midline.

Four training sets were extracted from the training set pool. The first two training sets included all 560 visual field records. The first set included the 25 test locations within the superior visual field and the second the 25 locations within the inferior visual field. The third and fourth training sets were composed of only those 560 field records with two or more than two missed locations (see below for definition of a missed location) within the respective hemifield. The training set for the superior hemifield was composed of 340 records while that for the inferior field was composed of 295 records.

PRE-PROCESSING OF FIELD DATA

The HVA threshold values were transformed into binary values (normal or defective). This transformation involved the computation of defect values (that is, the measured threshold minus the age matched expected threshold for a normal patient). The defect values were then adjusted for any shifts in overall sensitivity. The adjustment was based upon the difference between the 85 th percentile of ranked defect values from an individual field and the average 85th percentile from a group of normals ( 666 visual field records). The adjustment was limited to $2 \mathrm{~dB}$.

Once the pattern deviation values had been obtained a cut off criterion of $-6 \mathrm{~dB}$ was applied. Test locations with pattern deviation values of greater than and equal to $-6 \mathrm{~dB}$ were classified as normal while those with values below $-6 \mathrm{~dB}$ were classified as defective. This conversion process is similar to that used within the software package STATPAC which is designed to analyse visual field data from the HVA. ${ }^{16}{ }^{17}$ The binary input to the ANN means that the resultant classifications are based solely upon the spatial location and extent of the defects and not upon their depth.

\section{ARTIFICIAL NEURAL NETWORK}

The structure of the SOM network (Fig 2) can be conceived as a layer of input nodes $\mathrm{X}_{1}-\mathrm{X}_{\mathrm{m}}$

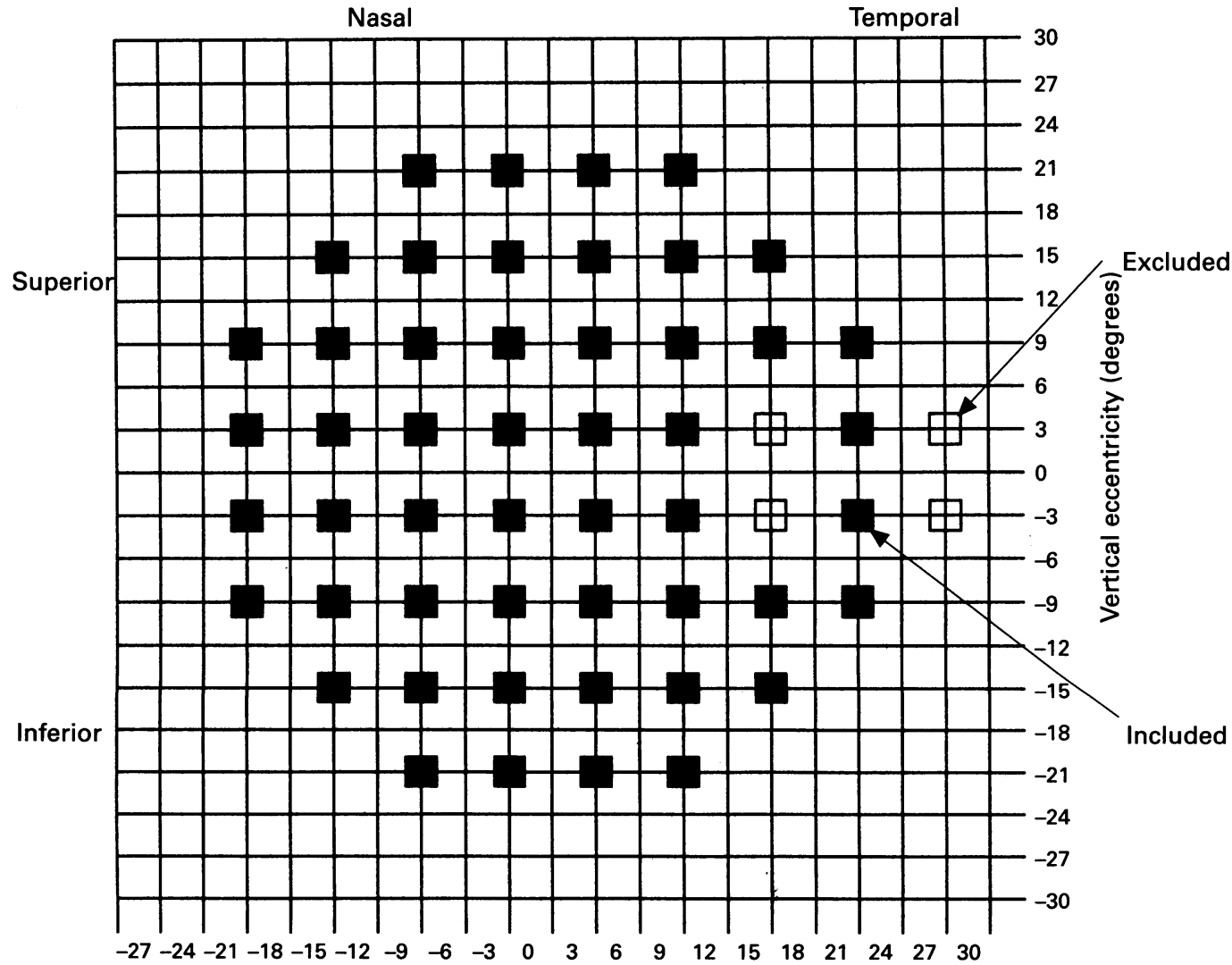

Temporal

Horizontal eccentricity (degrees

Figure 1 Stimulus locations of the Humphrey 24-2 program along with those selected for input into the artificial neural networks. 


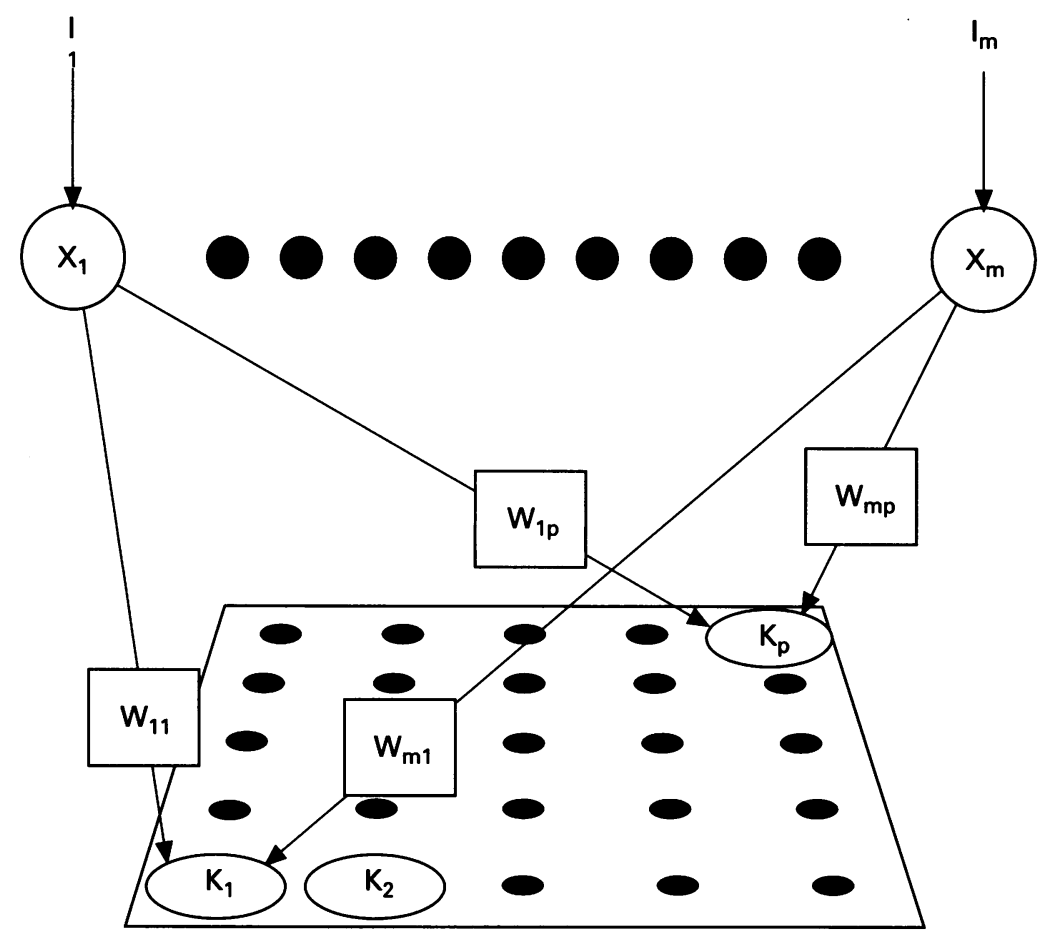

Figure 2 Schematic showing the structure of the Kohonen self organising feature map. Inputs $I_{1}-I_{m}$ correspond to the test locations, $K_{1}-K_{p}$ are the 25 output classes, while $W_{11}-W_{m p}$ represent the weights applied to each field location at each output class.

(each node corresponding to a test location within the visual field) fully connected to a two dimensional output layer (the map) of nodes $\mathrm{K}_{1}-\mathrm{K}_{\mathrm{p}}$. During training the weights associated with the connections between the input and output layers $W_{11}-W_{m p}$ are modified on the basis of the information contained within the visual field data and the training variables. After training a given visual field pattern will cause one output node to be more highly activated than the others. This node represents the spatial class of the defect. Kohonen networks were trained with NeuralWorks Professional II. ${ }^{18}$ Each network had 25 input nodes (one for each test location) and a 5 by 5 output layer. During training the network passed through the data set 30 times. A square neighbourhood function was used which began at the maximum dimension of the net (that is, 5) and decreased in size to just a single processing element while training progressed. This function determined which output nodes would have their weights modified during training and hence how ordered neighbourhoods develop upon the trained map.

\section{GRAPHICAL REPRESENTATION OF THE OUTPUT} NODES

Each of the 25 output nodes on the map effectively comes to represents a 'class' of visual field defect. This is graphically represented in this paper by the strength of the weights connecting each test location to the individual node. The strength of the weights is represented by a grey scale where dark areas represent strong weights (defect) and light areas represent weak weights (no defect). The 25 nodes/classes are numbered 0-24 from the top left to the bottom right; this numbering does not represent any characteristic of the data.

\section{Results}

The first two networks, those which were trained on the whole data set, are graphically presented in Figures 3 and 4. Normal eyes (those with no defective locations after transformation) are located within class 24 while the most severe defects are located in class 0 . Superior arcuate defects, which are a characteristic spatial pattern of loss associated with glaucoma, can be found in classes 15,16 , and 20 in Figure 3 while inferior arcuate defects can be found in classes 2 and 3 in Figure 4 .

In both maps several nodes have a total absence of any grey scale pattern. The weights for these nodes are in fact different but appear the same simply because they do not reach the cut off value for graphical representation. Adjusting the cut off levels to overcome this problem creates the reverse problem at the other end of the map, several nodes appearing to represent the same advanced defect pattern.

Networks trained on data in which there are two or more defective locations are graphically represented in Figures 5 and 6 . The most advanced defect patterns fall in class 0 while the most normal patterns fall in class 23 in Figure 5 and class 18 in Figure 6 . The absence of any visual fields with less than two defect locations within the training set has resulted in some major changes to the networks. There are now no nodes with a complete absence of any grey scaling and an increase in the number of nodes representing the more advanced defects. For example, in Figure 4 nodes 2 and 3 represent inferior arcuate defects extending from the blind spot. In Figure 6 nodes 3, 4, 8, and 12 represent the similar patterns of loss.

\section{Discussion}

Aulhorn and Harms ${ }^{1}$ subjectively listed eight classes of glaucomatous loss, five of which fell within the central region of the visual field. One of these central classes, enlargement of the blind spot, is not likely to be detected with a static technique whose spatial resolution is 6 degrees of visual angle. The other four classes (small scotoma, arcuate scotoma connected to blind spot, arcuate scotoma not connected to the blind spot, and ring scotoma) are all represented in the maps shown in Figures 3-6. In certain instances several nodes are allocated to a single Aulhorn and Harms class, the distinctions often being made on the basis of size and location. For example, Aulhorn and Harms class of small scotoma encompassed defects existing almost anywhere within the central field. In contrast, the ANN subdivides this class on the basis of defect location-a small scotoma in the superior nasal field being placed in a different class from a similar sized defect in the superior temporal field. The ANN has been configured to produce 25 classes of superior visual field loss and 25 classes of inferior visual field loss; this finer grading of spatial patterns of loss is useful for the monitoring of progressive defects.

It is possible to train SOMs with almost any number of output nodes. The choice of 25 nodes for both the superior and inferior field is based upon a consideration of a number of dif- 

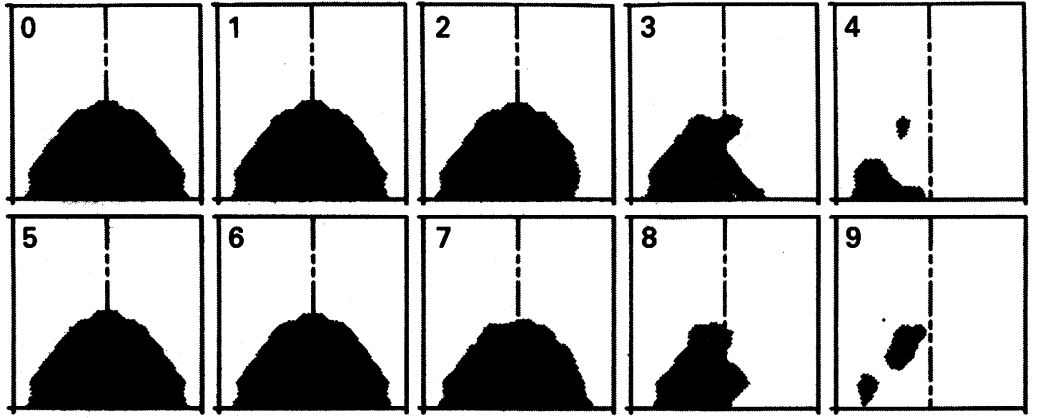

Weight

80 to 100

60 to 80

40 to 60

20 to 40
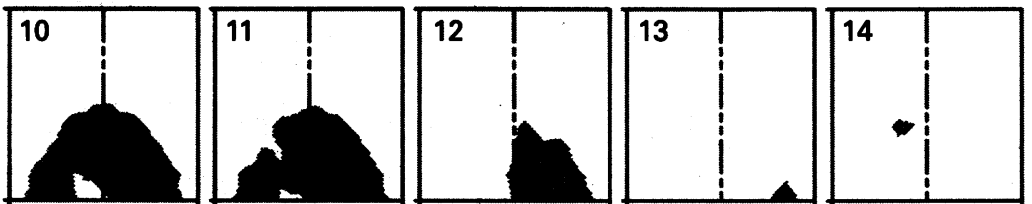

$\square 0$ to 20
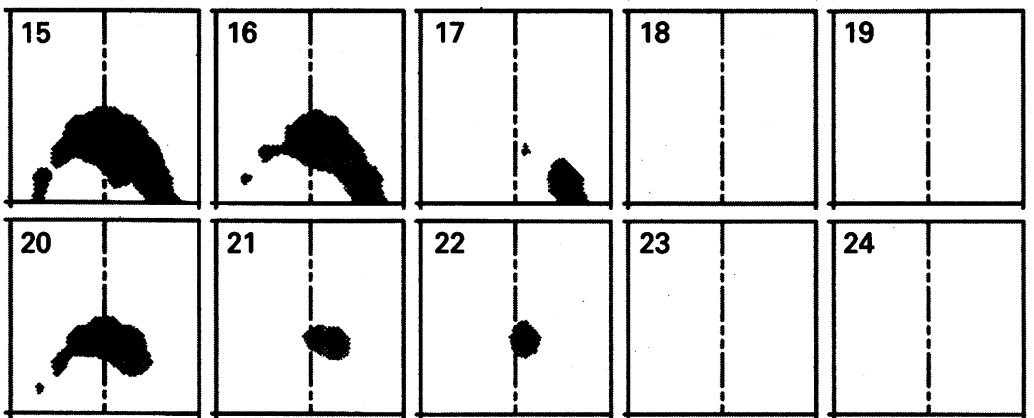

Figure 3 The 5 by 5 output map of a Kohonen network trained on 560 glaucomatous visual field defects. Training data came from 25 stimulus locations in the superior hemifield and included data from patients with both normal visual field and very early defects. Each grey scale represents the weights for each node on a scale ranging from 0 to 100 spatially organised to represent the visual field.
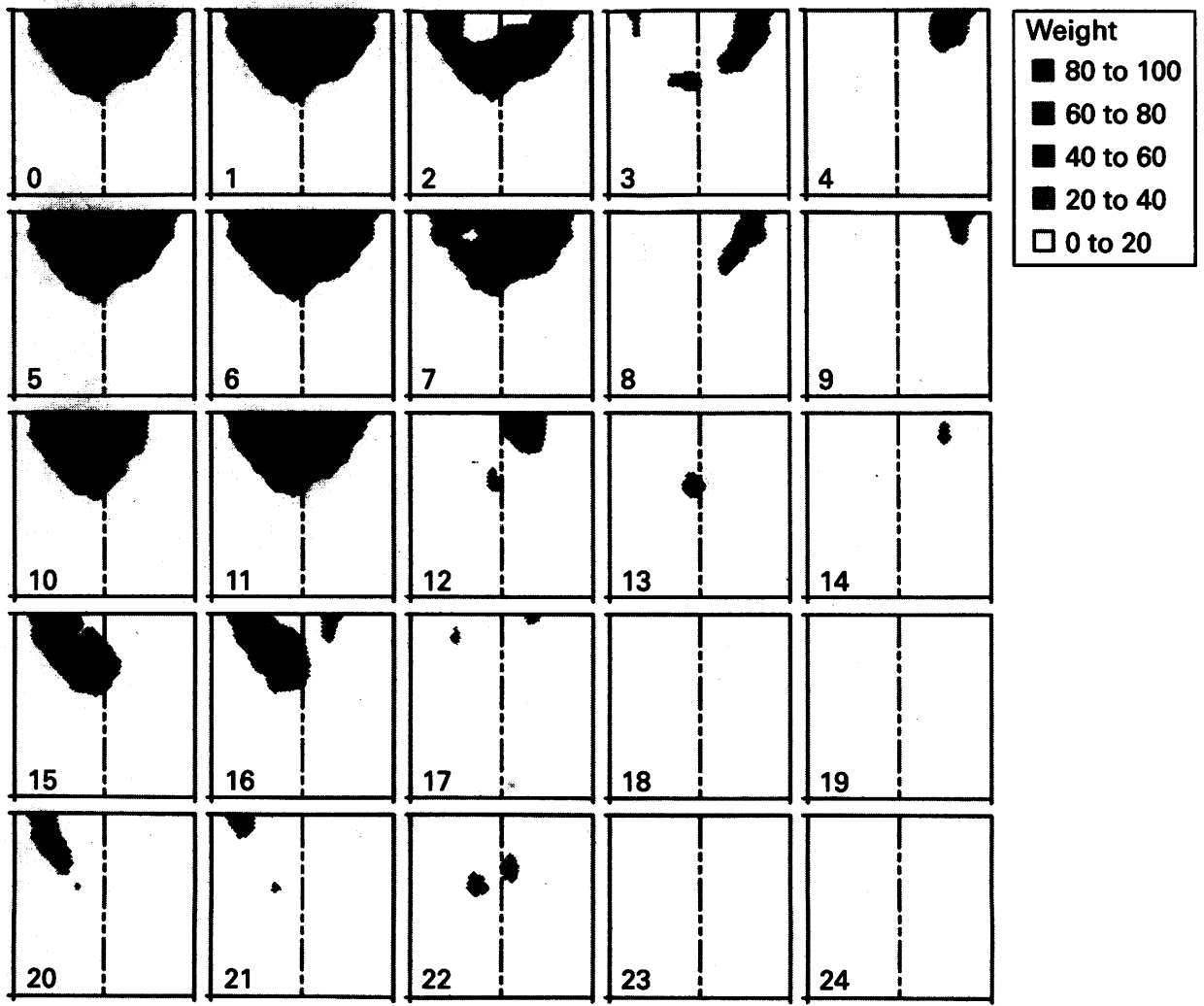

Figure 4 The 5 by 5 output map of Kohonen network trained on 560 glaucomatous visual field defects. Training data came from 25 stimulus locations in the inferior hemifield and included data from patients with both normal visual field and very early defects. Each grey scale represents the weights for each node on a scale ranging from 0 to 100 spatially organised to represent the visual field.

ferent factors. One of these is the likely number of classes that exist within the data. Training an SOM with more nodes than there are classes of data can result either in a number of redundant nodes (nodes whose weights are very similar to their neighbours but which are never actually activated) or in nodes which are specific to individual examples within the data. At the 

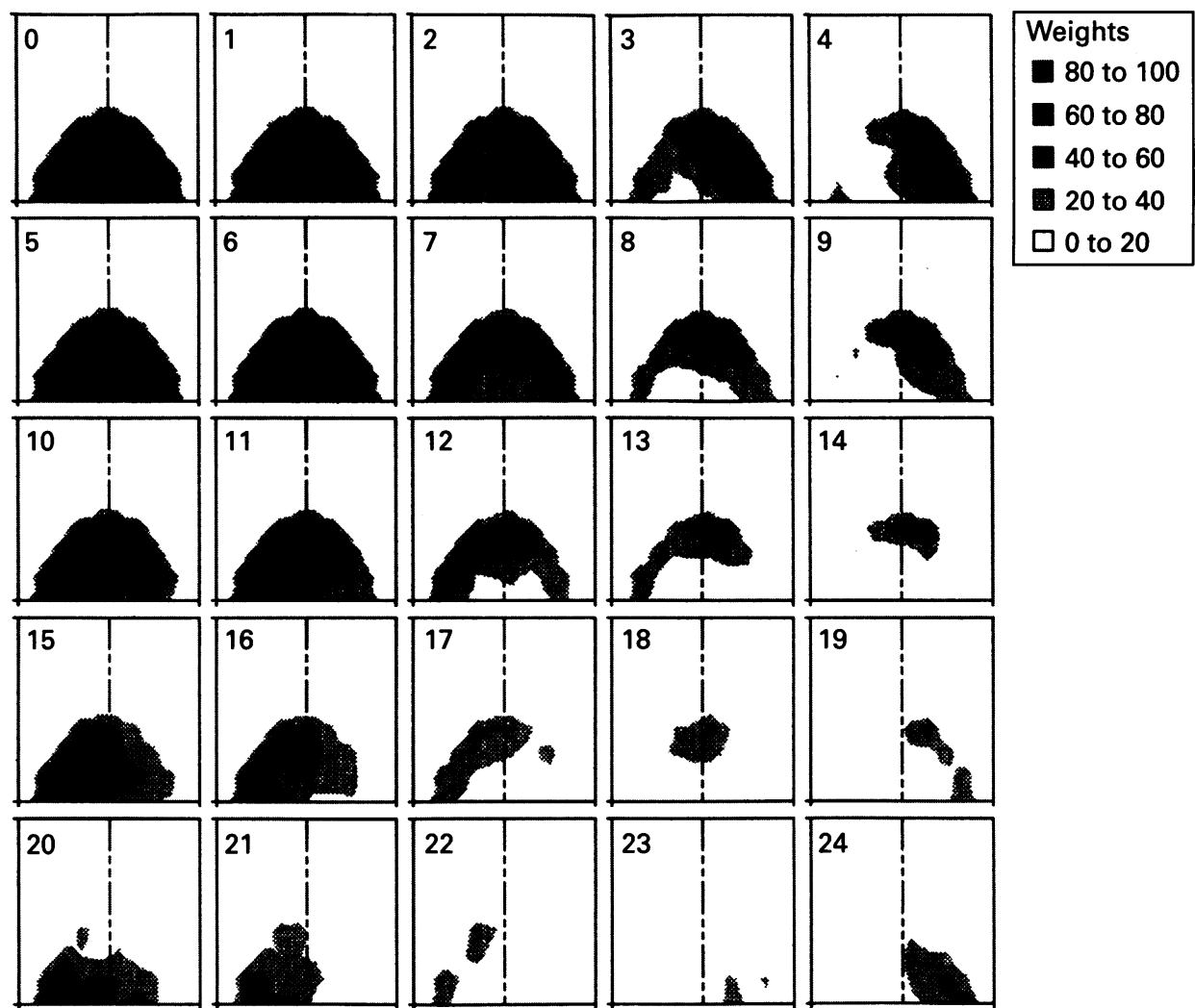

Figure 5 The 5 by 5 output map of Kohonen network trained on 340 glaucomatous visual field defects. Training data came from 25 stimulus locations in the superior hemifield. Training set inclusion criteria included missing two or more than two stimulus locations in the superior visual field. Each grey scale represents the weights for each node on a scale ranging from 0 to 100 spatially organised to represent the visual field.
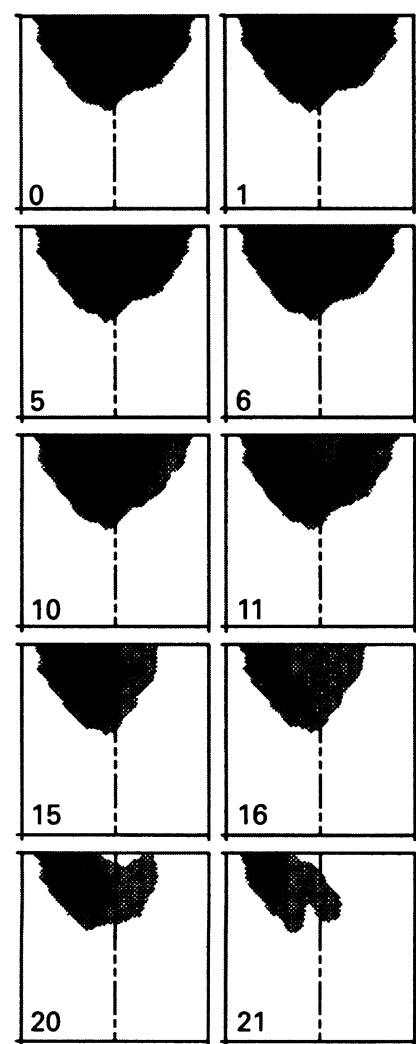
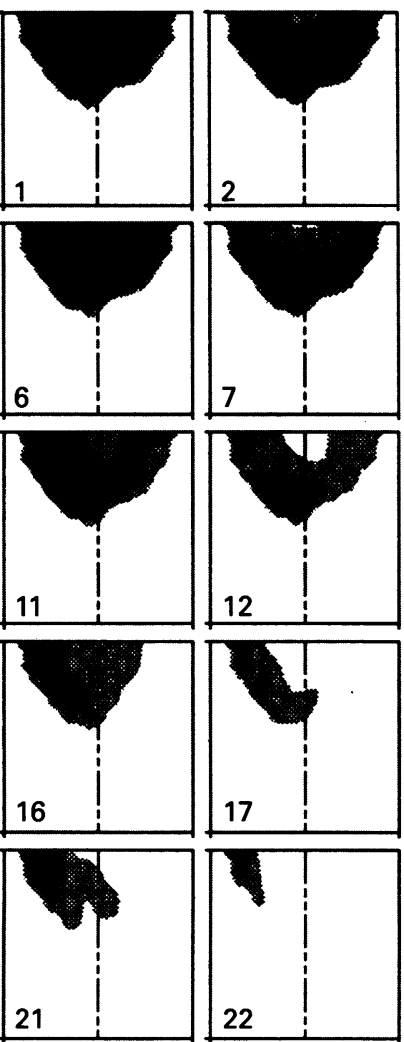
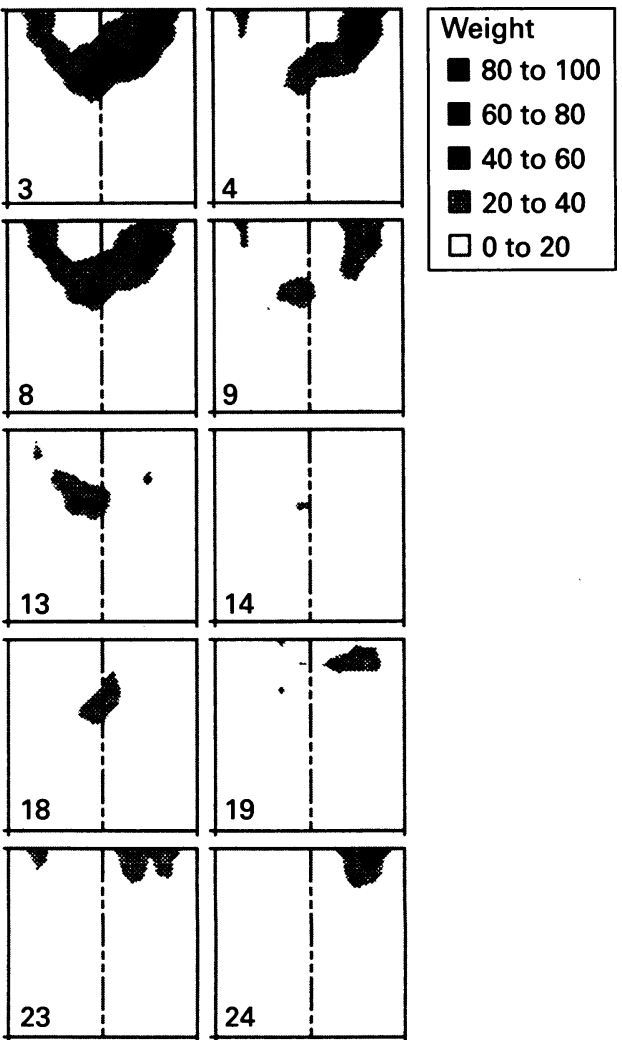

Figure 6 The 5 by 5 output map of Kohonen network trained on 295 glaucomatous visual field defects. Training data came from 25 stimulus locations in the inferior hemifield. Training set inclusion criteria included missing two or more than two stimulus locations in the inferior visual field. Each grey scale represents the weights for each node on a scale ranging from 0 to 100 spatially organised to represent the visual field.

other end of the scale, where the number of nodes are less than the number of classes, the network is forced to generalise, putting together similar classes and thereby limiting the discriminatory ability of the network. A second factor that needs to be taken into account when deciding upon the number of nodes within the network is the number of inputs. If 
there are only a few input nodes then the maximum number of potential (binary) patterns is also small. A third factor is the size of the training set. If the training set is small and the output map large then there is the danger that certain nodes will have very few examples and that these nodes will again represent specific examples rather than classes.

It is also possible to alter the form of the output map from the square shape used in this study to one which is either rectangular or linear. When deciding upon the appropriate form consideration needs to be given to the way in which defects progress and to the network's characteristic of trying to organise the outputs to form a continuum from one extreme to the other. The square form used in this research offers more options for smooth progression than would a rectangular or linear output map.

The map structure, which places early loss in one corner and advanced loss in the opposite corner, was derived by the network. At the onset of training there are random weights between each input and output node. As training progresses the extreme classes (early loss and advanced loss) gradually move towards extreme edges or corners of the map. The corners to which they move are influenced by the initial random settings of the weights, hence, re-training can produce maps which appear either rotated or flipped about an axis.

Maps trained with examples in which there are two or more than two defect locations do not have a node where the weights are all at or close to zero (no grey scale representation). When such maps are presented with data from a normal patient (no missed locations) they will generalise and place them in the 'most normal' class (class 23 in Fig 5 or class 18 in Fig 6). Hence, the maps produced in Figures 5 and 6 can be used to classify all visual fields and do not require a minimum of two missed locations.

The classifications of glaucomatous visual field defects found within this study are similar to those reported by Henson et al. ${ }^{19}$ This study used data obtained from the Henson CFS2000 perimeter using a suprathreshold strategy rather than the HVA full threshold program. The similarity of results suggests that the spatial patterns of loss are independent of the static strategy used.

The objective classification system developed by the ANN has several potential uses within clinical ophthalmology. It can be used to monitor the progression of visual field loss. In individual cases where a longitudinal series of visual field data is available each record can be classified and the path across the map examined to see if it moves in the direction of more advanced loss. Analysing groups of such patients may reveal characteristic paths which may in themselves be indicative of different forms of progression. The objective classification system can also be used to ensure that samples of patients are equivalent with respect to their spatial loss.
In summary, ANNs can be trained to classify objectively the spatial patterns of visual field loss found in glaucoma. These classifications, which are based solely upon the nature of the patterns of loss found within the training set, have several advantages over subjective ones. The ANNs include a precise definition of the class boundaries and hence are consistent in their classifications. They are capable of a finer classification than has hitherto been available with subjective techniques, which increases the potential of these networks to monitor glaucomatous loss. The ANN's output map shows a continuum of change when passing from one output class to its neighbour which enables the extent of loss to be quantified on the basis of its location with respect to least and most defective classes within the map.

Supported by the UK Engineering and Physical Sciences Research Council (grant number GR/H54393).

This work has been made possible by the kind contribution of visual field data from Mr R Hitchings and Dr B Chauhan. We visual field data from Mr R Hitchings and Dr B Chauhan. We
would also like to acknowledge the support of Dr F Fitzke, Dr w McNaught, and Mr D Crabb who helped in the selection of the data and in discussions of the work.

1 Aulhorn E, Harms $\mathrm{H}$. Early visual field defects in glaucoma. In: Leydhecker W, ed. Glaucoma. Tutzig symposium, 1966. Basel: Karger, 1967:151-75.

2 Aulhorn E. Comparative visual field study in patients with primary open angle glaucoma and anterior ischemic optic primary open angle glaucoma and anterior

3 Aulhorn E, Karmeyer H. Frequency distribution in early glaucomatous visual field defects. Doc Ophthalmol Proc Series 1977;14:75-83.

4 Drance SM. The early visual field defects in glaucoma. Invest Ophthalmol Vis Sci 1969;9:84-91.

5 Hart WM, Becker B. The onset and evolution of glaucomatous visual field defects. Ophthalmology 1982;89:268-79.

6 Jay JL, Murdoch JR. The rate of visual field loss in untreated primary open angle glaucoma. $\mathrm{Br} \mathcal{F}$ Ophthalmol 1993;77: 176-8.

7 Rumelhart DE, McClelland JL. Parallel distributed processing: explorations in the microstructure of cognition. Vol 1, Foundations. Cambridge, MA: MIT Press, 1986.

8 Goldbaum MH, Sample PA, White H, Weinreb RN. Discrimination of normal and glaucomatous visual fields by neural network. ARVO abstracts. Invest Ophthalmol Vis Sci 1990;31(4, suppl):503.

9 Shields SM, Trick GL. Applying neural networks in glaucoma: prediction of the risk of visual field loss. ARVO abstracts. Invest Ophthalmol Vis Sci 1990;31(4, suppl):503.

10 Kelman SE, Perell HF, Autrechy LD, Scott RJ. A neural network can differentiate glaucoma and optic neuropathy visual field through pattern recognition. In: Mills RP, Heijl A. eds. Perimetry update 1990/91. Amsterdam: Kugler A. eds. Perimetry update

11 Nagata $S$, Kani K, Sugiyama A. A computer assisted visual field diagnosis system using neural network. In: Mills RP, Heijl A. eds. Perimetry update 1990/91. Amsterdam: Kugler Publications, 1991:291-5.

12 Spenceley S, Henson DB, Bull DR. Visual field analysis using artificial neural networks. Ophthalmic Physiol Opt 1994;14:239-48.

13 Goldbaum MH, Sample PA, White H, Cote B, Raphaelian $\mathrm{P}$, Fechtner RD, et al. Interpretation of automated $\mathrm{P}$, Fechtner $\mathrm{RD}$, et al. Interpretation of automated perimetry for glaucoma by neur
molVis Sci 1994;35:3362-73.

14 Mutlukan E, Keating D. Visual field interpretation with a personal computer based neural network. Eye 1994;8: 321-3.

15 Kohonen T. Self-organization and associative memory. 2nd ed. New York: Springer-Verlag, 1988.

16 Heijl A, Lindgren G, Olsson J. A package for the statistical analysis of visual fields. Doc Ophthalmol Proc Ser 1987;49: $153-68$.

17 Heijl A, Lindgren G, Lindgren A, Olsson J, Asman P, Myers $S$, et al. Extended empirical statistical package for evaluation of single and multiple fields in glaucoma: Statpac 2. In: Mills RP, Heijl A, eds. Perimetry update 1990 91. Amsterdam: Kugler Publications, 1991: 303-15.

18 Neuralware. Neural computing: a technology handbook for professional II/plus and NeuralWorks explorer. Pittsburgh, PA: Neuralware Inc, 1993

19 Henson DB, Spenceley SE, Bull DR. Assessing progressive field loss in glaucoma using a self organising feature map. In: Mills RP, Wall M, eds. Perimetry update 1994/95. Amsterdam: Kugler Publications, 1995:1-11. 